\title{
Central Diabetes Insipidus after Transsphenoidal Surgery in Dogs with Cushing's Disease
}

\author{
Takahiro TESHIMA ${ }^{1) *}$, Yasushi HARA ${ }^{1)}$, Takahiro TAODA ${ }^{2)}$, Akira TERAMOTO $^{3)}$ and Masahiro TAGAWA ${ }^{1)}$ \\ ${ }^{1)}$ Division of Veterinary Surgery, Department of Veterinary Science, Faculty of Veterinary Medicine, Nippon Veterinary and Life Science \\ University 1-7-1 Kyonan-cho, Musashino-shi, Tokyo 180-8602, ${ }^{2}$ Department of Small Animal Surgery 2, School of Veterinary Medicine, \\ Kitasato University, 35-1 Higashi 23-bancho, Towada-shi, Aomori 034-8628 and ${ }^{3)}$ Department of Neurosurgery, Nippon Medical \\ School, 1-1-5 Sendagi, Bunkyo-ku, Tokyo 113-8603, Japan
}

(Received 26 March 2010/Accepted 6 August 2010/Published online in J-STAGE 20 August 2010)

\begin{abstract}
Transsphenoidal surgery (TSS) is an effective treatment for canine Cushing's disease, as well as human Cushing's disease. In humans, only the pituitary adenoma tissue is resected by TSS. However, in dogs, the whole pituitary including normal tissue is resected. Hence, central diabetes insipidus (CDI) may complicate the postoperative course in almost all dogs with Cushing's disease treated by TSS. However, it is difficult to assess the duration of the postoperative CDI, and whether it may be transient or permanent. In this study, postoperative CDI in 21 dogs with Cushing's disease and its predicted prognosis by preoperative parameters was investigated. In this study, CDI after TSS was classified as either transient or permanent based on the requirement for desmopressin. Preoperative circulating serum cortisol concentrations and pre- and post-operative plasma AVP concentrations were not significantly different between the transient CDI dogs and permanent CDI dogs. The duration of postoperative CDI was not correlated to the signal intensity ratio (posterior lobe of the pituitary/cerebral cortex), which is obtained from preoperative magnetic resonance imaging (MRI). However, the pituitary height/brain area $(\mathrm{P} / \mathrm{B})$ ratio of the permanent $\mathrm{CDI}$ dogs was significantly greater than that of the transient CDI dogs. In addition, there was a significant difference between the enlarged-pituitary $(\mathrm{P} / \mathrm{B}$ ratio $>0.31)$ transient $\mathrm{CDI} \operatorname{dogs}$ and the permanent $\mathrm{CDI}$ dogs. These results suggest that the incidence of postoperative permanent CDI is strongly influenced by the pituitary size. KEY WORDS: arginine vasopressin, dog, magnetic resonance image, pituitary tumor, signal intensity.
\end{abstract}

J. Vet. Med. Sci. 73(1): 33-39, 2011

Pituitary-dependent hyperadrenocorticism, or Cushing's disease, is a common endocrine disorder in dogs that is caused by an adrenocorticotropic hormone (ACTH) secreting corticotroph adenoma. The radical treatment for canine Cushing's disease is to resect the pituitary tumor by transsphenoidal surgery (TSS). Follow-up investigations of canine Cushing's disease treated by TSS have shown effective outcomes. Moreover, TSS leads to better results regarding survival and disease-free fractions than medical therapy in canine Cushing's disease $[12,13,16]$.

The major complications after TSS in dogs are hypernatremia and central diabetes insipidus (CDI) because the whole pituitary is resected $[13,21,24,25]$. Polydipsia and polyuria are extremely common signs associated with hyperadrenocorticism, and have been documented in approximately 80 to $85 \%$ of dogs with Cushing's disease [6]. Therefore, the duration of CDI and whether it is transient or permanent are common concerns of the owner. In previous studies, transient CDI normalizes 5 days after TSS in healthy dogs, but may persist up to 2 weeks in dogs with pituitary-dependent hyperadrenocorticism $[14,31]$. In addition, severe hypernatremia after TSS in dogs has been reported [21]. However, it is difficult to predict the degree of the postoperative CDI preoperatively.

In this study, we investigated postoperative CDI in 21

\footnotetext{
* Correspondence to: Dr. Teshima, T., Division of Veterinary Surgery, Nippon Veterinary and Life Science University, 1-7-1 Kyonan-cho, Musashino-shi, Tokyo 180-8602, Japan. e-mail: teshima@bg8.so-net.ne.jp
}

dogs with Cushing's disease, and whether it may be predicted with preoperative magnetic resonance images (MRI) and endocrinological parameters.

\section{MATERIALS AND METHODS}

Animals: Twenty one dogs with Cushing's disease that were treated by TSS and showed hypercortisolism that went into remission at follow-up at the Nippon Veterinary and Life Science University were included in the study [16]. The 21 dogs included 3 Beagle, 1 Bull Terrier, 2 Cavalier King Charles Spaniel, 1 Golden Retriever, 2 Miniature Dachshund, 1 Pembroke Welsh Corgi, 1 Shetland Sheepdog, 3 Shiba, 2 Yorkshire Terrier, and 5 cross breed dogs. There were 14 male dogs ( 6 castrated), and 7 female dogs (4 spayed). The median age at the time of surgery was 8.0 years (range, 4-11 years), and the median body weight was $11.1 \mathrm{~kg}$ (range, 5.8-33.4 kg) [Corticotroph adenoma (CA) group].

Cushing's disease was diagnosed by clinical signs, routine laboratory examination, endocrine examination (ACTH-stimulation test), abdominal ultrasonography, and MRI of the pituitary $[15,16,38,39]$. TSS was performed, and the resected pituitary tissues were diagnosed in each case as a corticotroph adenoma in the anterior lobe of the pituitary by immunohistochemical analysis, as described previously $[38,39]$. After TSS, postoperative hormone substitution therapy was performed as described previously [15, 24, 25]. Briefly, hydrocortisone (Hydrocortone, Banyu 
Pharmaceutical Co., Ltd., Japan) (1 mg/kg, i.v., TID) and desmopressin [Desmopressin (DDAVP), Kyowa Hakko Kogyo Co., Ltd., Japan) ] [(0.1-0.2 $\mu \mathrm{g} / \mathrm{kg}$, s.c. or $1-2$ drop $(1.25-2.5 \mu \mathrm{g} / \mathrm{head})$ into the conjunctival sac, SID-BID)] were administrated directly after removal of the pituitary gland. When the dogs had resumed drinking and eating, oral substitution therapy was started with prednisolone (Predonine, Shionogi \& Co., Ltd., Japan) (0.5 mg/kg, p.o., BID) and levothyroxine sodium (Thyradin-S, Aska Pharmaceutical Co., Ltd., Japan) (20 $\mu \mathrm{g} / \mathrm{kg}$, p.o., BID). Remission was defined as a basal serum cortisol concentration $<6 \mu \mathrm{g} / \mathrm{d} l$, a post-ACTH serum concentration $<17 \mu \mathrm{g} / \mathrm{d} l$, and a resolution of the clinical sign of hypercortisolism [16].

Twenty healthy Beagles having normal values for complete blood count, blood chemistry, blood gas analysis and serum electrolyte tests were used as the control group. There were 10 male dogs ( 2 castrated) and 10 female dogs (3 spayed). The median age was 1.8 years (range, $1-4$ years), and the median body weight was $10.2 \mathrm{~kg}$ (range, 8 $13 \mathrm{~kg}$ ) (control group).

Classification of central diabetes insipidus after TSS: CDI after TSS was classified based on the requirement for DDAVP administration. In this study, the requirement for DDAVP administration was defined as a urine specific gravity $\leq 1.005$, or plasma sodium concentrations $\geq 160$ $\mathrm{mEq} / l$. The permanent CDI group was defined as the requirement for desmopressin administration until death or until the latest available follow-up date. The transient CDI group was defined as discontinued DDAVP administration. Moreover, the transient CDI group was divided into enlarged transient CDI (the pituitary height/brain area $(\mathrm{P} / \mathrm{B})$ ratio $>0.31)$ and non-enlarged transient $\mathrm{CDI}(\mathrm{P} / \mathrm{B}$ ratio $\leq$ $0.31)$ groups.

Endocrine tests and hormone determination: An ACTHstimulation test was performed by collecting blood samples for measurement of the serum cortisol concentration at 0 and $60 \mathrm{~min}$ after the intravenous administration of $0.25 \mathrm{mg}$ of synthetic ACTH (Cortrosyn ${ }^{\circledR}$, Daiichi Sankyo, Tokyo, Japan) [11]. The serum cortisol concentrations were assayed as described previously [8]. All ACTH-stimulation tests were performed at 3 to $4 \mathrm{hr}$ after the administration of the morning hormone substitution therapy.

Plasma arginine vasopressin (AVP) concentrations were assayed using the radioimmunoassay (RIA) double antibody technique (AVP RIA Mitsubishi, Mitsubishi Kagaku Iatron, Tokyo, Japan), as described previously [37]. Before TSS, the blood samples for the assay were collected prior to MRI of the pituitary. All dogs in CA group were re-evaluated at $1,2,4,12$, and 24 weeks, and 1, 2, 3, and 4 years after TSS [16]. In the permanent CDI group, the plasma AVP concentration after TSS was assayed using the blood sample just prior to the administration of daily DDAVP acquired from the most recent re-evaluation. In the transient CDI group, the plasma AVP concentration after TSS was assayed from the blood sample acquired from the most recent re-evaluation.

Pituitary imaging and measurement of the Signal inten- sity ratio: MRI of the pituitary was performed using a 1.5 Tesla superconducting magnet (VISART, Toshiba Medicals, Tokyo, Japan) in all 21 dogs (CA group) before TSS. Food and water intake was withheld from all dogs for $12 \mathrm{hr}$ prior to MRI. T1-weighted transverse images were made before and after an intravenous bolus injection of $0.1 \mathrm{mmol}$ contrast medium $\left(\right.$ Omniscan $^{\circledR}$, gadodiamidehydrate $(\mathrm{Gd})$; Daiichi Sankyo, Tokyo, Japan) per kg body weight. With the dogs in sternal recumbence, transverse scans of the skull were made perpendicular to the skull base from the rostral clinoid processes to the dorsum sellae using the spin-echo method and a $350-\mathrm{ms}$ repetition time, a $15-\mathrm{ms}$ echo time, and $2.0-\mathrm{mm}$ thick consecutive slices. In the control group, the MRI of the pituitary was performed using the same methods as the CA group.

The pituitary height was measured on the image using the largest cross-section of the pituitary. From the height of the pituitary and the area of the brain obtained in the Gd T1weighted image, the $\mathrm{P} / \mathrm{B}$ ratio was calculated, as previously described [20]. Pituitaries with a $\mathrm{P} / \mathrm{B}$ ratio of more than 0.31 were considered to be enlarged.

The transverse images with the largest areas of the posterior lobe were used for measurement of the signal intensity (SI) ratio, as previously described [37]. SI was measured three times each at the posterior lobe and cerebral cortex from the transverse image, and the SI ratio was calculated from the transverse image as follows: SI ratio=average SI of the posterior lobe divided by average SI of the cerebral cortex.

Statistical analysis: Results are presented as mean \pm standard error for the data that were normally distributed (plasma AVP concentrations, serum cortisol concentrations, and SI ratio) and as median and ranges for data that were not normally distributed ( $\mathrm{P} / \mathrm{B}$ ratio). The plasma AVP concentrations and the SI ratio in the CA and control groups, and in the transient CDI and permanent CDI groups were compared by Student's $t$-test. Serum cortisol concentrations in the transient CDI and permanent CDI groups were compared by Student's $t$-test. P/B ratio in the transient and permanent CDI groups, and in the enlarged transient CDI, nonenlarged transient CDI, and permanent CDI groups were compared by two-sided Mann-Whitney's $U$ test and TukeyKramer post hoc test, respectively. Statistical analysis was performed using Excel 2003 with the add-in software Statcel 2. A difference with $P<0.05$ was considered significant.

\section{RESULTS}

Postoperative CDI: CDI appeared in all 21 dogs after TSS and was controlled by the administration of DDAVP. The transient CDI and permanent CDI groups consisted of 15 and 6 dogs, respectively. The transient CDI group included 11 male dogs (5 castrated) and 4 female dogs (2 spayed). The transient CDI group had a median age at the time of surgery was 8.0 years (range, 4-11 years), and a median body weight of $10.6 \mathrm{~kg}$ (range, $5.8-33.4 \mathrm{~kg}$ ). The enlarged and non-enlarged transient CDI groups consisted 
of 9 and 6 dogs, respectively. The permanent CDI group included 3 male dogs (1 castrated) and 3 female dogs (2 spayed), with a median age at the time of surgery of 7.0 years (range, 5-11 years), and a median body weight of 10.6 $\mathrm{kg}$ (range, 8.3-21.9 kg).

The period of DDAVP administration ranged from 3 to 30 days (median, 8 days) and 131 to 1428 days (4 dogs were still being administered DDAVP) in the transient CDI and permanent CDI groups, respectively.

The ACTH-stimulation test: The basal serum cortisol concentration before TSS in the transient CDI and permanent CDI groups was $10.2 \pm 1.6 \mu \mathrm{g} / \mathrm{d} l$ and $6.7 \pm 1.6 \mu \mathrm{g} / \mathrm{d} l$, respectively. The post-ACTH serum cortisol concentration before TSS in the transient CDI and permanent CDI groups was $49.3 \pm 3.6 \mu \mathrm{g} / \mathrm{d} l$ and $48.2 \pm 8.8 \mu \mathrm{g} / \mathrm{d} l$, respectively. No significant difference was observed in the basal serum cortisol concentration and post-ACTH serum cortisol concentration between the transient CDI and permanent CDI groups.

Plasma AVP concentration: In the control group, the plasma AVP concentration was $2.92 \pm 0.43 \mathrm{pg} / \mathrm{ml}$. The plasma AVP concentration before and after TSS in the CA group was $5.02 \pm 1.37 \mathrm{pg} / \mathrm{ml}$ and $0.96 \pm 0.19 \mathrm{pg} / \mathrm{ml}$, respectively. There was no significant difference between the control group and preoperative CA groups. The plasma AVP concentration in the control group was higher than that of postoperative CA group $(P<0.05)$ (Fig. 1).

The preoperative plasma AVP concentration in the transient CDI and permanent CDI groups was $5.49 \pm 1.90 \mathrm{pg} / \mathrm{m} l$ and $3.94 \pm 1.22 \mathrm{pg} / \mathrm{ml}$, respectively. The postoperative plasma AVP concentration in the transient CDI and permanent CDI groups was $1.04 \pm 0.26 \mathrm{pg} / \mathrm{m} l$ and $0.81 \pm 0.29 \mathrm{pg} /$ $\mathrm{m} l$, respectively. No significant difference was observed in the pre- and post-operative plasma AVP concentration between the transient CDI and permanent CDI groups (Fig. 1).

$P / B$ ratio: The median $\mathrm{P} / \mathrm{B}$ ratio in the transient $\mathrm{CDI}$ and permanent CDI groups was $0.33(0.24-0.47)$ and 0.46 (0.37-0.69), respectively. The median $\mathrm{P} / \mathrm{B}$ ratio in the nonenlarged transient CDI and enlarged transient CDI groups was $0.27(0.24-0.29)$ and $0.38(0.32-0.47)$, respectively. The $\mathrm{P} / \mathrm{B}$ ratio was significantly greater in the permanent CDI group than in the transient CDI group $(P<0.01)$ (Fig. 2). In addition, the $\mathrm{P} / \mathrm{B}$ ratio was significantly greater in the permanent CDI group than in the enlarged transient CDI group $(P<0.05)$ (Fig. 2).

SI ratio: The SI ratio in the control and CA groups was $2.22 \pm 0.04$ and $1.96 \pm 0.07$, respectively. The SI ratio in the transient and permanent groups was $2.00 \pm 0.08$ and $1.89 \pm$ 0.11 , respectively. The SI ratio was significantly greater in the control group than in the CA group $(P<0.01)$ (Fig. 3). However, there was no significant difference in the SI ratio between the transient CDI and permanent CDI groups (Fig. $3)$.

\section{DISCUSSION}

CDI is a polyuric syndrome that results from a lack of sufficient AVP to concentrate the urine for water conservation. In humans, only the pituitary adenoma tissue is selectively resected by TSS in the treatment of Cushing's disease. Transient CDI lasting 1-3 days occurs in as many as 30\% of patients after TSS $[3,17,31,36]$. Moreover, the incidence of permanent CDI is usually between 0.5 and $1.5 \%$ [2, 3, 17, $35,36,40]$. In contrast, in canine Cushing's disease, selective resection is difficult and the whole pituitary including normal tissue is resected. CDI may complicate the postoperative course in almost all dogs with Cushing's disease treated by TSS, because there is a sudden cessation of AVP secretion by the posterior lobe of pituitary after complete hypophysectomy [24]. DDAVP, a synthetic AVP analog, which is used for the treatment of postoperative CDI, has a strong affinity for vasopressin V2 receptors. In the renal
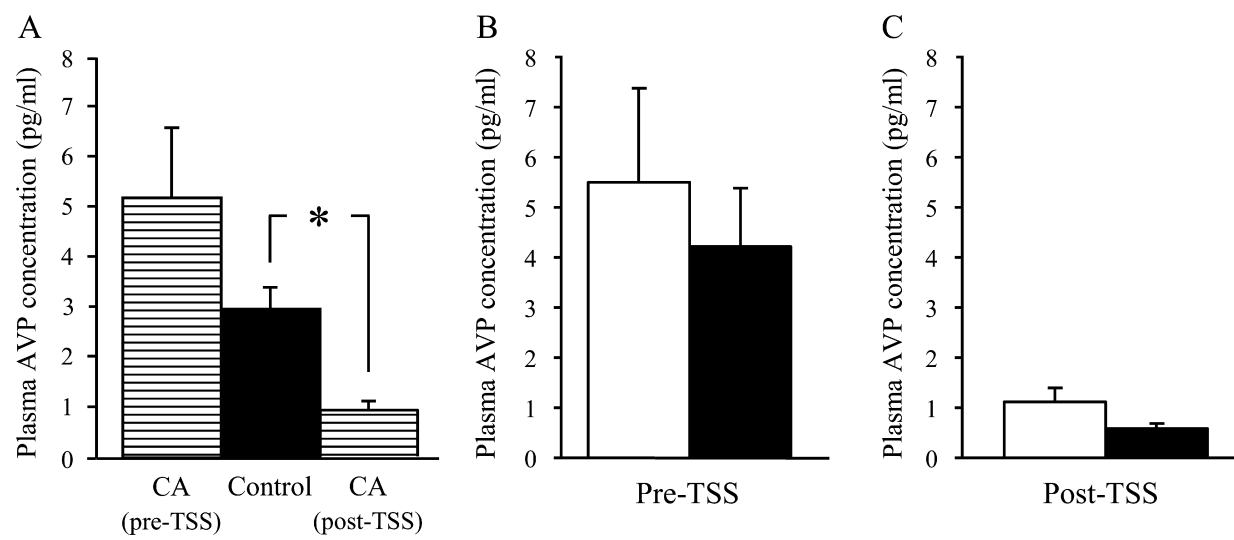

Fig. 1. Comparison of the plasma AVP concentration. TSS: transsphenoidal surgery. (A) The plasma arginine vasopressin (AVP) concentration in the control group was higher than that of postoperative (postTSS) corticotroph adenoma (CA) group. ${ }^{*}, P<0.05$ vs. control group. (B, C) Open bar: the transient central diabetes insipidus (CDI) group; closed bar, the permanent CDI group. No significant difference was observed in the pre- and post-TSS plasma AVP concentration between the transient CDI and permanent CDI groups. 

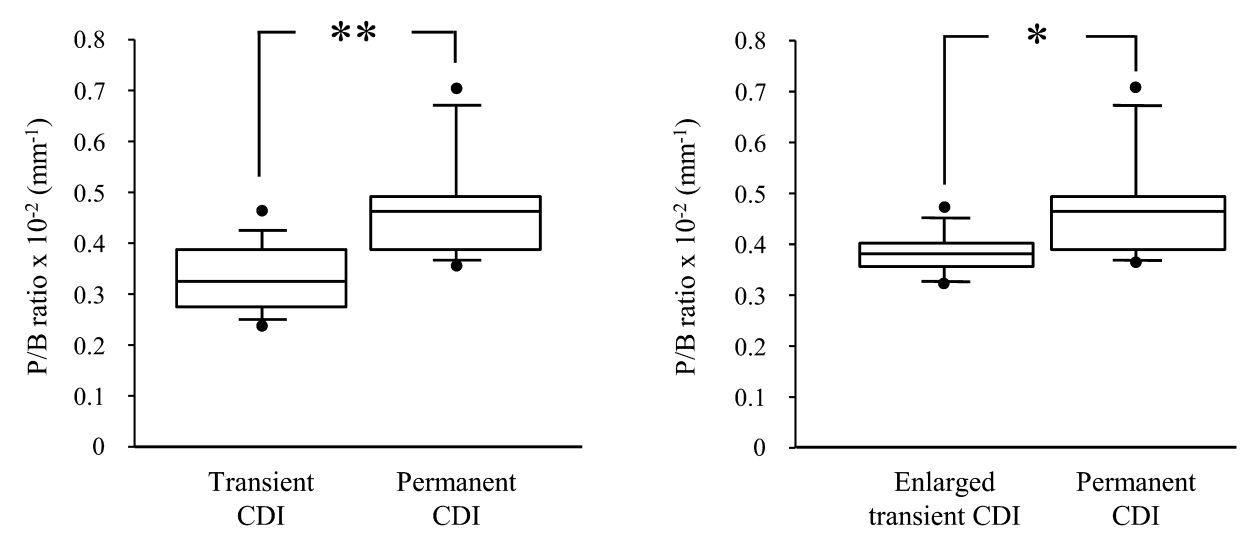

Fig. 2. Box-and-whisker plots of the $\mathrm{P} / \mathrm{B}$ ratio. Boxes represent values from the 25 th and 75 th percentiles. The horizontal line in each box is the median. Whiskers indicate the 10th and 90th percentiles, with any outlying data points marked. The pituitary height/brain area $(\mathrm{P} / \mathrm{B})$ ratio was significantly greater in the permanent central diabetes insipidus (CDI) group than in the transient CDI group (**, $P<0.01$ vs. transient CDI group). Moreover, the $\mathrm{P} / \mathrm{B}$ ratio of the permanent CDI group was significantly greater than that of the enlarged transient CDI group $(*, P<0.05$ vs. enlarged transient CDI group).
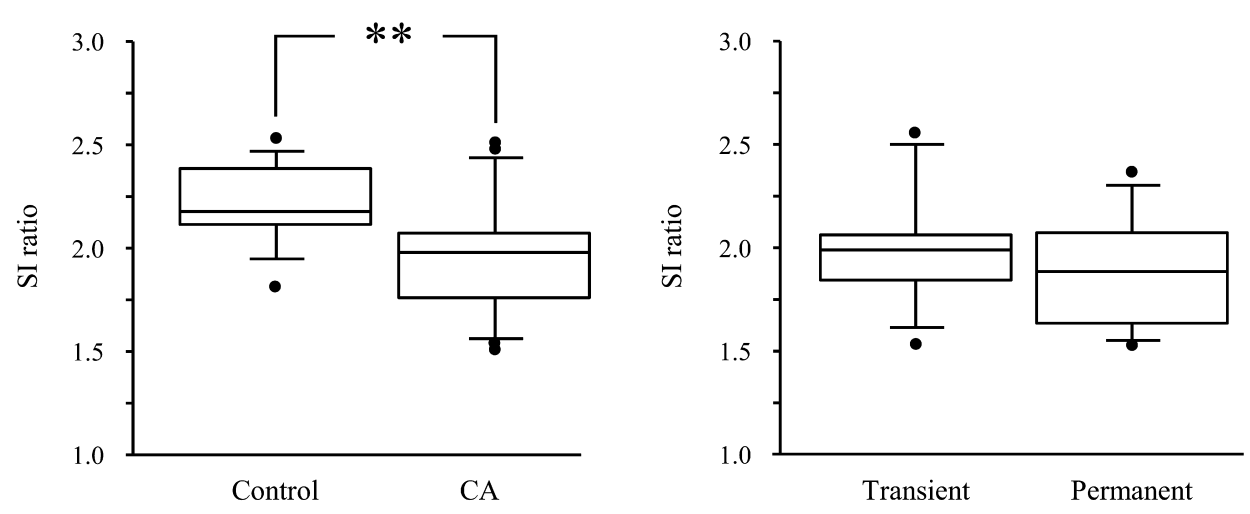

Fig. 3. Box-and-whisker plots of the SI ratio. Boxes represent values from the 25th and 75th percentiles. The horizontal line in each box is the median. Whiskers indicate the 10th and 90th percentiles, with any outlying data points marked. The signal intensity (SI) ratio was significantly greater in the control group than in the CA group (**, $P<0.01$ vs. control group).

collecting duct, water re-absorption is regulated by the antidiuretic hormone AVP. Binding of AVP to the vasopressin V2 receptor leads to the insertion of aquaporin-2 water channels in the apical membrane, thereby allowing water re-absorption from the pro-urine to the interstitium [7].

The modified water deprivation test or hypertonic saline infusion test are excellent tests to evaluate AVP secretion and the renal tubular response to AVP [7], however it is difficult to perform these tests postoperatively in dogs treated by TSS. In this study, we assessed whether DDAVP should be administrated to dogs after TSS based on the urine specific gravity $(\leq 1.005)$ and plasma sodium concentrations $(\geq$ $160 \mathrm{mEq} / l$ ) using the following criteria. Hypernatremia is observed frequently after TSS in dogs with Cushing's disease due to hyposthenuria caused by AVP deficiency. Hypernatremia is prevented by the administration of
DDAVP or regulated by dog itself to drink water as soon as possible after TSS. In the modified water deprivation test, the maximal secretion of AVP and concentration of urine are achieved when an animal loses 3 to $5 \%$ of body weight due to urine loss of fluid through urine with simultaneous water deprivation [7]. The urine specific gravity is less than 1.006 in most dogs with complete CDI, even if dehydrated and lose 3 to $5 \%$ of their body weight. Therefore, partial CDI dogs remained when we assessed the transient CDI dogs in the present study.

Glucocorticoids inhibit AVP release by a direct effect on the hypothalamus and/or neurohypophysis [28, 29]. This inhibition of AVP release is characterized by both an increase in osmotic threshold and a decrease in the sensitivity of the AVP response to increasing osmolality [1]. Hyperadrenocorticism also causes resistance to the effect of AVP in the kidney, possibly through interference with the 
action of AVP at the level of the renal collecting tubules or direct depression of renal tubular permeability to water. The results of the ACTH-stimulation test suggest that the duration of postoperative CDI was not influenced by the preoperative circulating cortisol concentrations. Therefore, the duration of postoperative CDI can not be predicted from the preoperative circulating cortisol concentrations.

Since the CDI persisted after TSS, pituitary tumor extension may occur in the dorsal direction due to an anatomically incomplete diaphragm of sella, and a prolonged mass effect by the tumor on the hypothalamic nuclei may have resulted in damage to AVP producing nuclei, such as the paraventricular and supraoptic nuclei, prior to TSS [13]. On the other hand, some authors have suggested that cortisol interference with the release of AVP is the most plausible explanation for polydipsia and polyuria, even in dogs with a large pituitary tumor $[7,30]$. In this study, no significant difference was observed in the preoperative plasma AVP concentration between the transient CDI and permanent CDI groups. This result suggests that direct compression of AVP producing nuclei by the pituitary tumor is rarely the cause of preoperative polyuria and polydipsia.

The posterior lobe of the normal pituitary shows characteristically high signal intensity in T1-weighted images at 2 months after birth or later in humans, which differs from the anterior lobe of the pituitary $[4,9]$. In addition, this characteristically high signal intensity is markedly decreased by the depletion of AVP in disorders such as CDI [10, 33, 34]. In a previous study, we concluded that the high signal intensity in the posterior lobe in T1-weighted images in normal dogs was caused by AVP stored at the site, and there was a significant correlation between the plasma AVP concentrations and the SI ratio in the posterior lobe [37]. In this study, the SI ratio was significantly lower in the CA group than in the control group. It has been suggested that the AVP storage in the posterior lobe decreased in dogs with Cushing's disease caused by polyuria and polydipsia. However, there was no difference in the SI ratio between the transient CDI and permanent CDI groups, and the duration of postoperative CDI can not be predicted from the SI ratio before TSS. The duration of postoperative CDI may be influenced by biological compensatory mechanisms, such as those involving the AVP receptor and aquaporin-2 water channel in the renal collecting duct, because no significant difference was observed between the plasma AVP concentrations before and after TSS in the transient CDI group and the permanent CDI group. It has been reported that in patients with CDI, long term deficiency of AVP increases the expression of vasopressin V2 receptors to maximum levels, which increases the sensitivity to AVP even at a low concentrations [22, 32]. Moreover, excess expression of the aquaporin-2 water channel in the collecting duct in a dehydration state may occur [5, 19].

In humans, although the postoperative CDI is transient in the overwhelming majority of cases, prolonged or permanent CDI, reportedly resulting from more proximal damage to the pituitary stalk and cell bodies in the hypothalamic nuclei, may also occur $[2,26]$. In dogs, when the pituitary stalk is sectioned low enough to avoid retrograde degeneration of supraoptic and paraventricular neurons, there is sufficient leakage of AVP to prevent diabetes insipidus [23]. In addition, the fibers of these magnocellular neurons may regenerate to establish new neurohemal connections and form a posterior pituitary-like structure. There is evidence that the meninges play an important role in this axonal regeneration process [18]. In our results, the $\mathrm{P} / \mathrm{B}$ ratio was significantly greater in the permanent CDI group than in the transient $\mathrm{CDI}$ group. Moreover, the $\mathrm{P} / \mathrm{B}$ ratio of the permanent CDI group was significantly greater than that of the enlarged transient CDI group. These results are similar to the previous report that the incidence of permanent CDI is significantly higher in dogs with a $\mathrm{P} / \mathrm{B}$ ratio $>0.31$ than in dogs with $\mathrm{P} / \mathrm{B}$ ratio $\leq 0.31$ [13]. We agree this author's comments that efforts to completely remove dorsally located tumor tissue in cases with large pituitary tumor may lead to damage of the median eminence, leading to more frequent CDI than in dogs with a small pituitary tumor. In humans, a risk factor for postoperative transient and permanent CDI is intraoperative cerebrospinal fluid (CSF) leak [26]. In this report, the author suggested that an intraoperative CSF leak may be a maker for a more aggressive resection involving more stalk manipulation. In contrast, all 21 dogs in CA group were observed intraoperative CSF leak from the third ventricle, because the whole pituitary including normal tissue was resected by TSS. Therefore, it is suggested that the intraoperative CSF leak can not be used a prognostic factor for the postoperative CDI in canine Cushing's disease. Interestingly, some authors have shown that postoperative CDI is more common after the resection of microadenomas [2, 14, 27]. This is because more extensive exploration of the gland and stalk may be required for successful resection of microadenomas, especially when a tumor is very small or does not appear on preoperative imaging. Increased manipulation of the stalk and gland may result in more damage and thus an increased risk of CDI. On the other hand, in patients with a macroadenoma, the tumor effectively displaces the posterior lobe of pituitary and the stalk upward, often leaving it covered with a fibrous layer. This location and protective layer may protect the posterior lobe of pituitary and may further explain the increased incidence of CDI after resection of a microadenoma $[3,27]$.

From our results, the risk of developing postoperative permanent CDI must be higher when the pituitary tumor is larger. The intraoperative damage to the pituitary stalk and hypothalamic nuclei may be influenced by the size of a pituitary tumor, because canine pituitary tumor extension almost occurs in the dorsal direction to the third ventricle. The postoperative plasma AVP concentration in the permanent CDI group decreased than in the transient group, however, there was no significant difference between the permanent CDI and transient CDI groups in this study. It may be due to including not sufficient cases in this study. Hence, it is not possible to conclude based on the present 
study, and re-evaluation should be necessary in the future.

In conclusion, prognostic factors for the duration of postoperative CDI were not identified in this study. However our result suggested that the incidence of permanent CDI is strongly influenced by the pituitary size, because the $\mathrm{P} / \mathrm{B}$ ratio of the permanent CDI group was significantly greater than that of the enlarged transient CDI group. Therefore, it is suggested that the risk of developing postoperative permanent CDI is higher, when dogs with larger pituitary tumors are treated by TSS.

\section{REFERENCES}

1. Biewenga, W. J., Rijnberk, A. and Mol, J. A. 1991. Osmoregulation of systemic vasopressin release during long-term glucocorticoid excess: a study in dogs with hyperadrenocorticism. Acta. Endocrinol. (Copenh) 124: 583-588.

2. Black, P. M., Zervas, N. T. and Candia, G. L. 1987. Incidence and management of complications of transsphenoidal operation for pituitary adenomas. Neurosurgery 20: 920-924.

3. Ciric, I., Ragin, A., Baumgartner, C. and Pierce, D. 1997. Complications of transsphenoidal surgery: results of a national survey, review of the literature, and personal experience. $\mathrm{Neu}$ rosurgery 40: $225-236$.

4. Cox, T. D. and Elster, A. D. 1991. Normal pituitary gland: changes in shape, size, and signal intensity during the 1st year of life at MR imaging. Radiology 179:721-724.

5. Deen, P. M., Verdijk, M. A., Knoers, N. V., Wieringa, B., Monnens, L. A., van Os, C. H. and van Oost, B. A. 1994. Requirement of human renal water channel aquaporin-2 for vasopressin-dependent concentration of urine. Science 264: 92-95.

6. Feldman, E. C. and Nelson, R. W. 2004. Canine hyperadrenocorticism (Cushing's syndrome). pp. 252-357. In: Canine and Feline Endocrinology and Reproduction, 3rd ed. (Feldman, E. C. and Nelson, R. W. eds.), WB Saunders, Philadelphia.

7. Feldman, E. C. and Nelson, R. W. 2004. Water metabolism and diabetes insipidus. pp. 2-44. In: Canine and Feline Endocrinology and Reproduction, 3rd ed. (Feldman, E. C. and Nelson, R. W. eds.), WB Saunders, Philadelphia.

8. Fracassi, F., Gandini, G., Diana, A., Preziosi, R., Ingh, T.S., Famigli-Bergamini, P. and Kooistra, H. S. 2007. Acromegaly due to a somatroph adenoma in a dog. Domest. Anim. Endocrinol. 32: 43-54.

9. Fujisawa, I., Asato, R., Nishimura, K., Togashi, K., Itoh, K., Nakano, Y., Itoh, H., Hashimoto, N., Takeuchi, J. and Torizuka, K. 1987. Anterior and posterior lobes of the pituitary gland: assessment by $1.5 \mathrm{~T}$ MR imaging. J. Comput. Assist. Tomogr. 11: 214-220.

10. Fujisawa, I., Nishimura, K., Asato, R., Togashi, K., Itoh, K., Noma, S., Kawamura, Y., Sago, T., Minami, S., Nakano, Y., Itoh, H. and Torizuka, K. 1987. Posterior lobe of the pituitary in diabetes insipidus: MR findings. J. Comput. Assist. Tomogr. 11: $221-225$.

11. Hansen, B. L., Kemppainen, R. J. and MacDonald, J. M. 1994. Synthetic ACTH (cosyntropin) stimulation tests in normal dogs: comparison of intravenous and intramuscular administration. J. Am. Anim. Hosp. Assoc. 30: 38-41.

12. Hanson, J. M., Teske, E., Voorhout, G., Galac, S., Kooistra, H. S. and Meij, B. P. 2007. Prognostic factors for outcome after transsphenoidal hypophysectomy in dogs with pituitary-dependent hyperadrenocorticism. J. Neurosurg. 107: 830-840.
13. Hanson, J. M., van't, H. M., Voorhout, G., Teske, E., Kooistra, H. S. and Meij, B. P. 2005. Efficacy of transsphenoidal hypophysectomy in treatment of dogs with pituitary-dependent hyperadrenocorticism. J. Vet. Intern. Med. 19: 687-694.

14. Hara, Y., Masuda, H., Taoda, T., Hasegawa, D., Fujita, Y., Nezu, Y. and Tagawa, M. 2003. Prophylactic efficacy of desmopressin acetate for diabetes insipidus after hypophysectomy in the dog. J. Vet. Med. Sci. 65: 17-22.

15. Hara, Y., Tagawa, M., Masuda, H., Sako, T., Koyama, H., Orima, H., Nakamura, S., Takahashi, K., Sanno, N. and Teramoto, A. 2003. Transsphenoidal hypophysectomy for four dogs with pituitary ACTH-producing adenoma. J. Vet. Med. Sci. 65: 801-804.

16. Hara, Y., Teshima, T., Taoda, T., Ishino, H., Nezu, Y., Harada, Y., Yogo, T., Masuda, H., Teramoto, A. and Tagawa, M. 2010. Efficacy of transsphenoidal surgery on endocrinological status and serum chemistry parameters in dogs with Cushing's disease. J. Vet. Med. Sci. 72: 397-404.

17. Hensen, J., Henig, A., Fahlbusch, R., Myer, M., Boehnert, M. and Buchfelder, M. 1999. Prevalence, predictors and patterns of postoperative polyuria and hyponatraemia in the immediate course after transsphenoidal surgery for pituitary adenomas. Clin. Endocrinol. (Oxf.) 50: 431-439.

18. Ishikawa, K., Kabeya, K., Shinoda, M., Katakai, K., Mori, M. and Tatemoto, K. 1995. Meninges play a neurotrophic role in the regeneration of vasopressin nerves after hypophysectomy. Brain Res. 677: 20-28.

19. Knepper, M. A. 1997. Molecular physiology of urinary concentrating mechanism: regulation of aquaporin water channels by vasopressin. Am. J. Physiol. 272: 3-12.

20. Kooistra, H. S., Voorhout, G., Mol, J. A. and Rijnberk, A. 1997. Correlation between impairment of glucocorticoid feedback and the size of the pituitary gland in dogs with pituitarydependent hyperadrenocorticism. J. Endocrinol. 152: 387-394.

21. Lantz, G. C., Ihle, S. L., Nelson, R. W., Carlton, W. W., Feldman, E. C., Lothrop, C. D. Jr. and Bottoms, G. D. 1988. Transsphenoidal hypophysectomy in the clinically normal dog. Am. J. Vet. Res. 49: 1134-1142.

22. Laycock, J. F. and Hanoune, J. 1998. From vasopressin receptor to water channel: intracellular traffic, constraint and bypass. J. Endocrinol. 159: 361-372.

23. Meij, B. P., Mol, J. A., van den Ingh, T. S., Bevers, M. M., Hazewinkel, H. A. and Rijnberk, A. 1997. Assessment of pituitary function after transsphenoidal hypophysectomy in beagle dogs. Domest. Anim. Endocrinol. 14: 81-97.

24. Meij, B. P., Voorhout, G., van den Ingh, T. S., Hazewinkel, H. A., Teske, E. and Rijnberk, A. 1998. Results of transsphenoidal hypophysectomy in 52 dogs with pituitary-dependent hyperadrenocorticism. Vet. Surg. 27: 246-261.

25. Meij, B. P., Voorhout, G., van den Ingh, T. S., Hazewinkel, H. A. and Van't Verlaat, J. W. 1997. Transsphenoidal hypophysectomy in beagle dogs: evaluation of a microsurgical technique. Vet. Surg. 26: 295-309.

26. Nemergut, E. C., Zuo, Z., Jane, J. A. Jr. and Laws, E. R. Jr. 2005. Predictors of diabetes insipidus after transsphenoidal surgery: a review of 881 patients. J. Neurosurg. 103: 448-454.

27. Olson, B. R., Gumowski, J., Rubino, D. and Oldfield, E. H. 1997. Pathophysiology of hyponatremia after transsphenoidal pituitary surgery. J. Neurosurg. 87: 499-507.

28. Papanek, P. E. and Raff, H. 1994. Chronic physiological increases in cortisol inhibit the vasopressin response to hypertonicity in conscious dogs. Am. J. Physiol. 267: 1342-1349.

29. Papanek, P. E., Sladek, C. D. and Raff, H. 1997. Corticosterone 
inhibition of osmotically stimulated vasopressin from hypothalamic-neurohypophysial explants. Am. J. Physiol. 272: 158162.

30. Raff, H. 1987. Glucocorticoid inhibition of neurohypophysial vasopressin secretion. Am. J. Physiol. 252: 635-644.

31. Rees, D. A., Hanna, F. W., Davies, J. S., Mills, R. G., Vafidis, J. and Scanlon, M. F. 2002. Long-term follow-up results of transsphenoidal surgery for Cushing's disease in a single centre using strict criteria for remission. Clin. Endocrinol. (Oxf.) 56: 541-551.

32. Richardson, D. W. and Robinson, A. G. 1985. Desmopressin. Ann. Intern. Med. 103: 228-239.

33. Sato, N., Endo, K., Ishizaka, H. and Matsumoto, M. 1993. Serial MR intensity changes of the posterior pituitary in a patient with anorexia nervosa, high serum $\mathrm{ADH}$, and oliguria. J. Comput. Assist. Tomogr. 17: 648-650.

34. Sato, N., Ishizaka, H., Yagi, H., Matsumoto, M. and Endo, K. 1993. Posterior lobe of the pituitary in diabetes insipidus: dynamic MR imaging. Radiology. 186: 357-360.

35. Semple, P. L. and Laws, E. R. Jr. 1999. Complications in a contemporary series of patients who underwent transsphenoidal surgery for Cushing's disease. J. Neurosurg. 91: 175-179.
36. Singer, P. A. and Sevilla, L. J. 2003. Postoperative endocrine management of pituitary tumors. Neurosurg. Clin. N. Am. 14: 123-138.

37. Teshima, T., Hara, Y., Masuda, H., Taoda T., Nezu, Y., Harada, Y., Yogo, T., Hasegawa, D., Orima, H., Osamura, R. Y. and Tagawa, M. 2008. Relationship between Arginine Vasopressin and High Signal Intensity in the Pituitary Posterior Lobe on T1-Weighted MR Images in Dogs. J. Vet. Med. Sci. 70: 693-699.

38. Teshima, T., Hara, Y., Shigihara, K., Takekoshi, S., Nezu, Y., Harada, Y., Yogo, T., Teramoto, A., Osamura, R.Y. and Tagawa, M. 2009. Coexistence of corticotroph adenoma and thyrotroph hyperplasia in a dog. J. Vet. Med. Sci. 71: 93-98.

39. Teshima, T., Hara, Y., Taoda, T., Koyama, H., Takahashi, K., Nezu, Y., Harada, Y., Yogo, T., Nishida, K., Osamura, R.Y. and Teramoto, A. and Tagawa, M. 2008. Cushing's disease complicated with thrombosis in a dog. J. Vet. Med. Sci. 70: 487-491.

40. Wilson, C. B. and Dempsey, L. C. 1978. Transsphenoidal microsurgical removal of 250 pituitary adenomas. J. Neurosurg. 48: 13-22. 